\title{
Measuring recognition memory in zebrafish larvae: issues and limitations
}

\author{
Matteo Bruzzone ${ }^{\text {Equal first author, } 1}{ }^{\text {, Elia Gatto }}{ }^{\text {Corresp., Equal first author, } 1}{ }^{1}$, Tyrone Lucon Xiccato ${ }^{2}$, Luisa Dalla Valle ${ }^{3}$, Camilla Maria \\ Fontana $^{3}$, Giacomo Meneghetti ${ }^{3}$, Angelo Bisazza ${ }^{1,4}$ \\ 1 Department of General Psychology, University of Padova, Padova, Italy \\ 2 Department of Life Sciences and Biotechnology, University of Ferrara, Ferrara, Italy \\ 3 Department of Biology, University of Padova, Padova, Italy \\ 4 Padova Neuroscience Center, University of Padova, Padova, Italy \\ Corresponding Author: Elia Gatto \\ Email address: elia.gatto@phd.unipd.it
}

Recognition memory is the capacity to recognize previously encountered objects, events or places. This ability is crucial for many fitness-related activities, and it appears very early in the development of several species. In the laboratory, recognition memory is most often investigated using the novel object recognition test (NORt), which exploits the tendency of most vertebrates to explore novel objects over familiar ones. Despite the use of larval zebrafish is rapidly increasing in research on brain, cognition and neuropathologies, it is unknown whether larvae possess recognition memory and whether the NORt can be used to assess it. Here, we tested a NOR procedure in zebrafish larvae of 7-, 14- and 21-days post-fertilization (dpf) to investigate when recognition memory first appears during ontogeny. Overall, we found that larvae explored a novel stimulus longer than a familiar one. This response was fully significant only for 14-dpf larvae. A control experiment evidenced that larvae become neophobic at 21-dpf, which may explain the poor performance at this age. The preference for the novel stimulus was also affected by the type of stimulus, being significant with tri-dimensional objects varying in shape and bidimensional geometrical figures but not with objects differing in colour. Further analyses suggest that lack of effect for objects with different colours was due to spontaneous preference for one colour. This study highlights the presence of recognition memory in zebrafish larvae but also revealed non-cognitive factors that may hinder the application of NORt paradigms in the early developmental stages of zebrafish. 
1 Measuring recognition memory in zebrafish larvae: issues and limitations

2 Matteo Bruzzone ${ }^{1}$, Elia Gatto ${ }^{1}$, Tyrone Lucon-Xiccato ${ }^{2}$, Luisa Dalla Valle ${ }^{3}$, Camilla Maria

3 Fontana $^{3}$, Giacomo Meneghetti ${ }^{3}$ and Angelo Bisazza ${ }^{1,4}$

4 1. Department of General Psychology, University of Padova, Padova, Italy

5 2. Department of Life Sciences and Biotechnology, University of Ferrara, Ferrara, Italy

6 3. Department of Biology, University of Padova, Padova, Italy

7 4. Padova Neuroscience Center, University of Padova, Padova, Italy

8 Corresponding Author:

$9 \quad$ Elia Gatto

10 Department of General Psychology, University of Padova, Padova, Italy

11 Email address: elia.gatto@phd.unipd.it 


\section{ABSTRACT}

13 Recognition memory is the capacity to recognize previously encountered objects, events or

14 places. This ability is crucial for many fitness-related activities, and it appears very early in the

15 development of several species. In the laboratory, recognition memory is most often investigated

16 using the novel object recognition test (NORt), which exploits the tendency of most vertebrates

17 to explore novel objects over familiar ones. Despite the use of larval zebrafish is rapidly

18 increasing in research on brain, cognition and neuropathologies, it is unknown whether larvae

19 possess recognition memory and whether the NORt can be used to assess it. Here, we tested a

20 NOR procedure in zebrafish larvae of 7-, 14- and 21-days post-fertilization (dpf) to investigate

21 when recognition memory first appears during ontogeny. Overall, we found that larvae explored

22 a novel stimulus longer than a familiar one. This response was fully significant only for 14-dpf

23 larvae. A control experiment evidenced that larvae become neophobic at 21-dpf, which may

24 explain the poor performance at this age. The preference for the novel stimulus was also affected

25 by the type of stimulus, being significant with tri-dimensional objects varying in shape and bi-

26 dimensional geometrical figures but not with objects differing in colour. Further analyses suggest

27 that lack of effect for objects with different colours was due to spontaneous preference for one

28 colour. This study highlights the presence of recognition memory in zebrafish larvae but also

29 revealed non-cognitive factors that may hinder the application of NORt paradigms in the early

30 developmental stages of zebrafish.

31

32 Keywords: recognition memory, zebrafish larvae, NOR test, neophobia 


\section{INTRODUCTION}

35 The ability to learn and memorize the characteristics of objects and to recognize an object when

36 it is encountered again is of fundamental importance for many fitness-related activities, such as

37 obtaining food, avoiding predators or interacting with conspecifics. Recognition memory allows

38 one to discriminate familiar stimuli from novel ones and to adjust one's behaviour accordingly

39 (Antunes \& Biala, 2012; Blaser \& Heiser, 2015). In mammals and birds, recognition memory

40 seems to appear very early. Researchers have observed recognition memory in pre-weaning rats,

41 and they have reported the preference for novel objects as early as the first week of an infant's

42 life (Johnson \& Horn, 1986; Pascalis, 1994; Reger, Hoyda \& Giza, 2009). The same pattern

43 may occur in fish as well. Five-day-old guppies (Poecilia reticulata) can discriminate a familiar

44 object from an unfamiliar one (Miletto Petrazzini et al., 2012). However, the reproductive mode

45 of the guppy is atypical for fishes, as it is a livebearer. At birth, the fry is fully developed and

46 endowed with a complex behavioural repertoire and a suite of cognitive abilities not much

47 different from those of adults (Magurran \& Seghers, 1990; Bisazza et al., 2010; Miletto

48 Petrazzini et al., 2012; Piffer, Miletto Petrazzini \& Agrillo, 2013).

49 Many other species of fish do not present such an advanced stage of development at birth. For

50 example, the zebrafish, which is the main teleost model for translational research, has a very

51 short embryonal development and hatches at 2-3-days after fertilization. The nervous system of

52 zebrafish larvae is poorly developed (Nusslein-Volhard \& Dahm, 2002). They can swim, but

53 they do not feed autonomously until the sixth-day post fertilization (dpf) and do develop some

54 form of social behaviour only from $21 \mathrm{dpf}$ (Wilson, 2012; Hinz \& de Polavieja, 2017). There is

55 little information available on the cognitive abilities of larval zebrafish. Valente and colleagues

56 (2012) showed that zebrafish larvae can be conditioned to avoid one side of the tank by 
57 repeatedly pairing this position with electroshock. Learning started from approximately $21 \mathrm{dpf}$,

58 and adult performance was reached around $42 \mathrm{dpf}$. Another study demonstrated the possibility of

59 conditioning the tail contraction in 6- to 8-dpf zebrafish after repeatedly pairing a moving spot of

60 light with a touch of the larva's body (Aizenberg \& Schuman, 2011). However, it is unknown

61 whether zebrafish larvae memorize the features of the objects that they encounter and whether

62 they can discriminate amongst different objects.

63 Memory research with animal models was greatly boosted by the introduction of a new paradigm

64 in 1988, the novel object recognition test (NORt), which is a simple and fast procedure to

65 measure recognition memory in rats (Ennaceur \& Delacour, 1988; Antunes \& Biala, 2012).

66 Concisely, a rat is allowed to briefly explore a new object introduced in its cage, and, after a

67 temporal interval, the same object is again introduced in the cage, paired with a novel one. Rats

68 tend to ignore the familiar object, and the relative time spent exploring the novel object is taken

69 as a measure of recognition memory. The NOR test is particularly amenable to comparative and

70 development studies, as it is based on the spontaneous tendency towards tactile or visual

71 exploration of novel over familiar stimuli, and the task appears to be less affected by potential

72 confounds due to contingency rules, as well as by potential stress components, due to long

73 training procedures (reviewed in Blaser \& Heyser, 2015). Researchers have used the NORt

74 paradigm and its variants to study recognition memory in a variety of mammals and birds

75 (Kornum et al., 2007; Ennaceur, 2010; Barnes, Burke \& Ryan, 2012; Soto \& Wasserman, 2014),

76 and more recently they have attempted to use it to study memory in zebrafish using this method

77 (Gerlai, 2016). Lucon-Xiccato and Dadda (2014) applied a variant of the original NORt to study

78 object recognition in zebrafish. In the familiarization phase, fish were familiarized with one

79 object for $25 \mathrm{~min}$. When subjects were exposed in the test phase to the familiar and a novel 
80 object, they spent more time near the novel stimulus. Using similar procedures, other studies

81 found that zebrafish can identify the movement and direction of virtual geometrical shapes

82 (Braida et al., 2014) and discriminate objects based on colour and shape (Oliveira et al., 2015;

83 May et al., 2016) or the previous location of the familiarized object (Hamilton et al., 2016). To

84 date, no attempt has been made to study when these important functions emerge and how they

85 develop during ontogeny.

86 The scope of our study was to determine whether larval zebrafish display some form of

87 recognition memory, when this ability first appears in development and whether there is a

88 reliable procedure to measure it. Most research on zebrafish nervous system, including those on

89 neurodevelopmental and neurodegenerative disorders, is performed in larvae (Bandmann \&

90 Burton, 2010; Sager, Bai \& Burton, 2010; Sakai, Ijaz \& Hoffman, 2018). Larvae can be obtained

91 in great number, favoring large-scale screenings of drugs and genotypes (Norto, 2013;

92 Richendrfer et al., 2012), and their synaptic activation can be monitored in vivo at the whole-

93 brain resolution (Leung et al., 2013). Among the others, zebrafish models for the re-myelination

94 process in multiple sclerosis and in brain injuries (Buckley, Goldsmith \& Franklin, 2008), and for

95 alterations in TAU-protein function in pathologies such as Alzheimer's disease (Paquet et al.,

96 2009) have been developed in larvae. These and other applications of zebrafish larvae may

97 greatly benefit from efficient methods to measure memory in the early developmental stages.

98 Zebrafish are generally considered larvae between 3 and 29 dpf. However, before 6 dpf, their

99 visual system does not fully respond to visual stimuli (Huang \& Neuhauss, 2008). In our

100 experiments, we investigated zebrafish of three ages, which were expected to allow describing

101 developmental trajectories of recognition memory of larvae: 7-, 14- and 21-dpf. Experiments 1

102 and 2 aimed to assess the ability of larvae to remember familiar objects differing in colour or 
103 shape. We adapted the classical NORt paradigm developed for rats (Ennaceur \& Delacour,

104 1988). During the familiarization phase, each larva was exposed to two identical objects

105 (experiment 1b: small cubes of the same colour, either red or green; experiment 2: objects of the 106 same colour but with a different shape, cube vs. cone). During the testing phase, the subject was 107 confronted with one familiar object and one of the objects of either a new colour (experiment 1b) 108 or the new shape (experiment 2). We measured the time spent near the familiar or the novel 109 object. Before performing the NOR experiment based on objects with different colour, we 110 assessed the presence of spontaneous colour preferences in larvae. This was done to identify 111 colours that cause innate avoidance or attraction and could impact the results of the NOR 112 experiment. The presence of colour preference is well documented in adult zebrafish (Spence et 113 al, 2008; Avdesh et al., 2012), but results of studies in larvae are contrasting (Park et al., 2016;

114 Peeters, Moeskops \& Veenvliet, 2016). In experiment 3, we performed a variant of the 115 recognition test using two bi-dimensional printed geometrical figures. Indeed, some studies have 116 adopted bi-dimensional stimuli to study visual recognition in zebrafish and other species (Braida 117 et al., 2013; Braida et al., 2014) and these stimuli can be easily modified to produce several 118 variants. To better interpret the results of our previous experiments, in experiment 4 we studied 119 ontogenetic changes in the tendency of larvae to approach unfamiliar objects (i.e., neophobia).

120 We positioned subjects in a test apparatus where an unfamiliar object (a black cone) was present 121 and measured the tendency to approach it.

123 MATERIAL \& METHODS

124 Ethical statement 
125 The experiments adhere to the current legislation of our country (Decreto Legislativo 4 Marzo

126 2014, n. 26) and were approved by the Ethical Committee of University of Padova (OPBA

127 18/2018, protocol n. 159333 - 30/03/2018).

128

129 Subject

130 The subjects were wild-type zebrafish larvae of three different ages: 7-, 14- and 21-day post-

131 fertilization (dpf) obtained by spawning from a strain maintained in our laboratory and originally

132 bought in a local pet shop. Larvae were housed in Petri dishes $(10 \mathrm{~cm} \emptyset, \mathrm{h}: 1.5 \mathrm{~cm})$ in a solution

133 of Fish Water 1x (0.5mM NaH2PO4 * H2O, 0.5mM Na2HPO4 * H2O, 1.5gr Instant Ocean, 11

134 de-ionized H2O) and Methylene blue $(0.0016 \mathrm{gr} / 1)$ at a density of approximately 30 individuals

135 each. The illumination was set on a 14:10 h light:dark cycle and the temperature was maintained

136 at $28.5 \pm 1^{\circ} \mathrm{C}$. Larvae were fed two times a day with dry food (particle size: $0.75 \mathrm{~mm}$ ) from the

137 age of $6 \mathrm{dpf}$.

138 We planned to test 40 zebrafish in experiment 1a for each age (120 larvae in total), 20 in

139 experiment $1 \mathrm{~b}$ for each age (60 larvae in total), 20 in experiment 2 for each age (60 larvae in

140 total), 20 in experiment 3 for each age (60 larvae in total) and 20 in experiment 4 for each age

141 (60 larvae in total). Forty zebrafish (8 subjects for experiment 1a, 13 subjects for experiment $1 \mathrm{~b}$,

1426 subjects for experiment 2, 2 subjects for experiment 3 and 11 subjects for experiment 4) were

143 discarded and substituted with new subjects to maintain the predetermined sample size (see

144 details below). The overall study included 360 subjects that completed the four experiments, plus

145 the 40 zebrafish discarded (total: 400).

146

147 Experiment 1a: Colour Preference 
148 We used a setup similar to one previously used for studying spontaneous colour preference in

149 adult zebrafish (Oliveira et al., 2015). The experimental apparatus (Fig. 1A) consisted of a Petri

150 dish filled with $1 \mathrm{~cm}$ of Fish Water at $28.5 \pm 1^{\circ} \mathrm{C}$. To prevent the possible effects of external

151 cues, the apparatus was placed in the centre of an empty, white room with uniform illumination.

152 The water was changed at every trial. The wall and bottom of the petri dish presented LEGO $^{\circledR}$

153 bricks of four different colours, namely blue (RGB: 0, 61, 165), green (RGB: 0, 173, 69), yellow

154 (RGB: 255, 237, 0) and red (RGB: 227, 0, 11). The LEGO $^{\circledR}$ bricks subdivided the platform into

155 four equivalent sectors. We built the apparatus with $\mathrm{LEGO}^{\circledR}$ bricks as the stimuli used for the

156 following four experiments. We used three different colour combinations: (clockwise) red -

157 green - blue - yellow; red - blue - yellow - green; red - yellow - green - blue. The orientation

158 of the colours was also rotated across subjects. At the centre of the platform, a grey plastic

159 square $(1 \times 1 \mathrm{~cm})$ was used as the starting point during the test. The apparatus was illuminated

160 by a $30-W$ fluorescent lamp. A Canon LEGRIA HFR38 was positioned at $90 \mathrm{~cm}$ above the

161 apparatus for video recording. Each subject was individually inserted in the centre of the

162 apparatus. The behaviour was recorded for $10 \mathrm{~min}$.

163

164 Experiment 1b: Novel Object Recognition Test (Object's Colour)

165 The experimental apparatus (Fig. 1B) consisted of a Petri dish $(10 \mathrm{~cm} \emptyset, \mathrm{h}: 1.5 \mathrm{~cm})$ filled with 1 $166 \mathrm{~cm}$ of Fish Water. The walls of the apparatus were covered with white paper to prevent the fish

167 from seeing the surrounding room. The apparatus was illuminated by a $30-\mathrm{W}$ fluorescent lamp,

168 and the temperature was maintained at $28.5 \pm 1{ }^{\circ} \mathrm{C}$. A Canon LEGRIA HFR38 was positioned at $16990 \mathrm{~cm}$ for video recording. Stimuli consisted of plastic cubes $\left(1 \times 1 \mathrm{~cm}, \mathrm{LEGO}^{\circledR} \mathrm{ID:}\right.$ : 3005$)$ of

170 two different colours. Based on the result of experiment 1a, larvae showed a similar preference 
171 for green and red sectors built with LEGO $^{\circledR}$ bricks. We chose these two colours for the stimuli.

172 The familiarization phase lasted for 3 days. On the evening of the first day, subjects were

173 individually introduced to the experimental apparatus with the stimuli already present. During

174 the familiarization phase, the two presented objects were identical, either two red or two green

175 LEGO $^{\circledR}$ cubes. The colour of the stimulus was counterbalanced amongst the subjects. Three

176 times a day, with a 4-h interval, both objects were removed for $10 \mathrm{~min}$ and then positioned again

177 in the apparatus, in a different position. This changing was aimed to familiarize the subjects to

178 the disappearance and reappearance of the objects. On the morning of the fourth day, subjects

179 were fed 1 -h before the test. The test consisted of removing the two identical LEGO ${ }^{\circledR}$ cubes and,

180 after $10 \mathrm{~min}$, replacing them with two $\mathrm{LEGO}^{\circledR}$ cubes that differed in colour. We presented one

181 identical $\mathrm{LEGO}^{\circledR}$ cube that the subjects were familiar with and a novel one the subjects had not

182 yet experienced. The position of the familiar and novel stimulus was randomly varied across

183 subjects. The exploratory behaviour was recorded for $8 \mathrm{~min}$.

184

185 Experiment 2: Novel Object Recognition Test (Object's Shape)

186 Apparatus and procedure were the same for the previous experiment 1b, except for the fact that 187 the stimuli were two LEGO $^{\circledR}$ bricks of the same colour but different shape. They were a green 188 LEGO $^{\circledR}$ cubes $\left(1 \times 1 \mathrm{~cm}\right.$; LEGO ${ }^{\circledR}$, ID: 3005$)$ and a green cone $\left(1 \times 1 \mathrm{~cm}\right.$; LEGO ${ }^{\circledR}$, ID: 59900; 189 Fig. 1C).

190

191 Experiment 3: Recognition of Bi-Dimensional Geometrical Figure

192 We used two different apparatuses for the familiarization phase and the test phase. The apparatus 193 used to habituate the subjects was made of a single Plexiglas tank of $22 \times 10 \times 12 \mathrm{~cm}$, and it was 
194 filled with $6 \mathrm{~cm}$ of Fish Water 1x. Stimuli were black bi-dimensional geometrical figures made 195 with Microsoft PowerPoint and printed on white paper with a laser printer. Stimuli were placed 196 along the outer sides of the short walls of the familiarization tank (Fig. 1D). The long walls of 197 the familiarization tank were covered with white paper to prevent the fish from seeing the room. 198 The test apparatus (Fig. 1D) consisted of a single Plexiglas tank $(8 \times 4 \times 5 \mathrm{~cm})$ filled with $2.5 \mathrm{~cm}$ 199 of Fish Water $(80 \mathrm{~mL})$. The temperature was maintained at $28.5 \pm 1^{\circ} \mathrm{C}$. The bottom and the long 200 sides of the test tank were covered by white paper to prevent external interference.

201 Familiarization and test apparatus were illuminated by a 30-W fluorescent lamp. A Canon 202 LEGRIA HFR38 was positioned at $90 \mathrm{~cm}$ above the test apparatus for video recording. The 203 stimuli were presented on the shorter side of the test apparatus. Stimuli consisted of a black 204 circle geometrical figure $\left(0.62 \mathrm{~cm} \emptyset\right.$, area: $\left.0.3 \mathrm{~cm}^{2}\right)$ and a black triangle geometrical figure (1: $2050.86 \mathrm{~cm}$, h: $0.72 \mathrm{~cm}$, area: $0.3 \mathrm{~cm}^{2}$ ). The position of the stimuli was counterbalanced between the 206 shorter sides of the test apparatus. The familiarization phase lasted 4 days. In the morning of the 207 first day, subjects were introduced to the familiarization tank where one out of two bi208 dimensional geometrical figures were presented (either a circle or a triangle). On the fifth day, 209 each subject was individually inserted in the test apparatus presenting both the familiar 210 geometrical shape and the novel one. The exploratory behaviour was recorded for $12 \mathrm{~min}$.

\section{Experiment 4: Development of Neophobia}

213 The test apparatus (Fig. 1E) consisted of a Plexiglas tank $(8 \times 4 \times 5 \mathrm{~cm})$ filled with $2.5 \mathrm{~cm}$ of 214 Fish water $1 \mathrm{x}(80 \mathrm{~mL})$. The test apparatus was covered with white paper up to $4 \mathrm{~cm}$. The 215 apparatus was illuminated by two 30-W fluorescent lamps. The stimulus consisted of a black 216 cone (diameter base: $0.7 \mathrm{~cm}, \mathrm{~h}: 1 \mathrm{~cm}$ ) placed over a white pedestal $(\mathrm{h}: 1.3 \mathrm{~cm})$. The position of 
217 the stimulus was counterbalanced across subjects between the shorter sides of the test apparatus.

218 A Canon LEGRIA HFR38 was placed $90 \mathrm{~cm}$ above the experimental apparatus for video

219 recording. Subjects were individually inserted at the centre of the unoccupied half of the test

220 apparatus. The exploratory behaviour was recorded for $10 \mathrm{~min}$.

221

\section{Analysis of the recordings}

223 We analysed the performance of subjects from the digital recordings played back on a computer

224 screen. The recordings were coded in Advanced Video Codec High Definition format, at 25

225 frames per seconds and high resolution (1920 x 1080 pixels), allowing precise spatial and

226 temporal resolution of larvae position. To analyse the exploratory behaviour of the stimuli, we

227 virtually considered specific sectors of the experimental apparatus according to each experiment.

228 In experiment 1a, we divided the test apparatus into four equivalent sectors in correspondence

229 with the colour. In experiments $1 \mathrm{~b}$ and 2, we considered a circular area $(\varnothing: 2.0 \mathrm{~cm})$ around each

230 stimulus. In experiment 3 , we divided the apparatus into four equivalent sectors $(2 \times 4 \mathrm{~cm})$, and

231 we only considered the area close to the stimuli. In experiment 4, we divided the apparatus into

232 two equivalent sectors. We analysed the video recordings using the software BORIS (Behavioral

233 Observation Research Interactive Software; University of Torino, Torino, Italy) by a blind

234 experimenter. The software calculated the time spent in each sector of apparatus. As a measure

235 of preference for the coloured sector (experiment 1a), preference for the novel object

236 (experiments 1b, 2 and 3) or preference for the unfamiliar object (experiment 4), we computed

237 the proportion of time close to the reference stimulus over the total time spent close to both

238 stimuli (experiments 1b, 2 and 3) or sectors (experiments 1a and 4). 


\section{Statistical analysis}

241 We performed the statistical analysis in RStudio version 1.1.383 (RStudio Team (2015).

242 RStudio: Integrated Development for R. RStudio, Inc., Boston, MA URL The statistical tests

243 were two-tailed, and the significance threshold was $p=0.05$. Descriptive statistics are reported

244 as mean \pm standard deviation. http://www.rstudio.com/). Data were checked for normality before

245 the analysis using the Kolmogorov-Smirnov test. Because the proportion of time spent in each

246 coloured sectors in experiments 1a did not show a normal distribution, we performed an arcsine

247 square-root transformation. Data presented two outliers (one 21-dpf larvae in experiment 1b, and

248 one 14-dpf larva in experiment 3 ) consisting of subjects that spent $80 \%$ more close to one

249 stimulus due to the less activity compared with the average of the other subjects. Because

250 diagnostic plots showed that these two outliers substantially reduced models' fit, we dropped

251 them from the datasets. We analysed the time spent close to both stimuli and the proportion of

252 time close to the reference stimulus (see details above) using ANOVA to evaluate differences

253 amongst factors. We fit models with the considered independent variables, ages as the fixed

254 factor in all five experiments and the coloured sectors as the fixed factor only in experiment 1a,

255 as well as the stimulus used to the familiarization phase in experiments $1 \mathrm{~b}, 2$ and 3 . When we

256 found a significant difference between ages, we computed a Tukey post-hoc analysis to evaluate

257 the differences between the levels of this factor. Then, we compared the proportion of time close

258 to the referenced stimulus for each age by performing a one-sample two-tailed t-test against the

259 chance level (50\%).

260 In experiments $1 \mathrm{a}$ and 4 , we analysed the temporal pattern of larvae preferences using a linear

261 mixed-effects model (LMMs, 'Imer' function of the 'Ime4' R package) fitted with the minutes as

262 the covariate, age as the fixed factor and subject ID as the random effect to account for repeated 
263 measures. For experiment 1a, we also fitted the LMM with the colour of the sectors as the fixed 264 factor. We evaluated the effect of these factors using 'Anova' function of the 'ImerTest' 265 package.

266 In the meta-analysis of time spent near the stimuli, we initially normalized the data of the three 267 NORt experiments (Z-score). The transformed data were then analysed with ANOVA fitted with 268 the age and experiment as factors.

269

270

\section{RESULTS}

\section{Experiment 1a: Colour Preference}

272 Subjects spent a different amount of time in the four sectors $\left(F_{3,468}=35.753, p<0.001, \eta p^{2}=\right.$ 273 0.186), and there was no difference amongst the three ages $\left(F_{2,468}=0.349, p=0.705, \eta p^{2}=\right.$ $2740.001)$, interaction $\left(F_{6,468}=0.476, p=0.826, \eta p^{2}=0.006\right.$; Fig. 2A). A Tukey post-hoc test 275 indicates that subjects spent significantly more time in the blue sector $(192.01 \pm 107.77 \mathrm{~s}$, mean $276 \pm \mathrm{SD})$ compared to the green $(88.19 \pm 38.02 \mathrm{~s} ; p<0.001)$, red $(99.38 \pm 69.47 \mathrm{~s} ; p<0.001)$ and 277 yellow sectors $(99.85 \pm 80.61 \mathrm{~s} ; p<0.001)$, but there was no difference amongst the green, red 278 and yellow sectors (all $p>0.5$ ). Adding the factor "minutes of the test" to the model reveals that 279 preference for blue significantly decreases with time (Fig. $2 \mathrm{~B}, \mathrm{C}, \mathrm{D})$ : $\operatorname{sector}\left(\chi^{2}{ }_{3}=418.559, p<\right.$ $\left.2800.001, \eta p^{2}=0.075\right)$, interaction between "minutes of the test" and sector $\left(\chi^{2}{ }_{3}=98.130, p<\right.$ $\left.2810.001, \eta p^{2}=0.025\right)$, and interaction between age and sector $\left(\chi^{2}{ }_{6}=13.312, p=0.038, \eta p^{2}=\right.$ 2820.003 ). No other factors or interactions were significant (all $p>0.06$ ). 
285 In the test phase, subjects, as a whole, spent $139.68 \pm 79.79 \mathrm{~s}(29.10 \pm 16.62 \%)$ close to one or

286 the other object, and there was no difference amongst the three ages $\left(F_{2,57}=3.046, p=0.055, \eta p^{2}\right.$

$287=0.097$ ). Larvae did not show a preference for the familiar or the novel stimulus (percentage of

288 time spent close to the novel stimulus $51.96 \pm 25.56 \%$; one sample $\mathrm{t}$ test: $t_{58}=0.588, p=0.559$,

289 Cohen's $d=0.077$; Fig. 3A). There was no difference amongst ages $\left(F_{2,53}=0.124, p=0.884\right.$,

$\left.290 \eta p^{2}=0.005\right)$ or for the colour of stimulus used during the familiarization phase $\left(F_{1,53}=2.511, p\right.$

$\left.291=0.119, \eta p^{2}=0.045\right)$, interaction $\left(F_{2,53}=0.106, p=0.203, \eta p^{2}=0.058\right)$.

292 A separate analysis of the three ages showed that no age group showed a preference for the novel

293 or the familiar stimulus (percentage of time spent close to the novel stimulus in 7-dpf larvae

$29451.57 \pm 19.86 \%$; one sample t test: $t_{19}=0.353, p=0.728$, Cohen's $d=0.079 ; 14$-dpf: $54.09 \pm$

$29529.95 \%, t_{19}=0.611, p=0.548$, Cohen's $d=0.137 ; 21$-dpf: $50.12 \pm 27.09 \%, t_{18}=0.019, p=$

296 0.985, Cohen's $d=0.004)$.

297

298

\section{Experiment 2: Novel Object Recognition Test (Object's Shape)}

299 In the test phase, subjects, as a whole, spent $189.67 \pm 58.75 \mathrm{~s}(39.52 \pm 14.41 \%)$ close to one or

300 the other object, and there was no difference amongst the three ages $\left(F_{2,57}=0.767, p=0.469, \eta p^{2}\right.$

$301=0.026$ ). Larvae showed a significant preference for the novel stimulus (percentage of time spent

302 close to the novel stimulus: $55.88 \pm 21.98 \% ; t_{59}=2.073, p=0.043$, Cohen's $d=0.268$, Fig. 3B).

303 There was no difference amongst ages $\left(F_{2,54}=0.961, p=0.389, \eta p^{2}=0.034\right)$ or in the shape of

304 stimulus used in the familiarization phase $\left(F_{1,54}=1.085, p=0.302, \eta p^{2}=0.020\right)$; the interaction

305 between these two factors was significant $\left(F_{2,54}=4.761, p=0.013, \eta p^{2}=0.150\right)$. To understand

306 this significant interaction, we performed a split analysis split for age and a split analysis for the

307 stimulus used for familiarization. 
308 The shape of stimulus used for familiarization had a significant effect in 7-dpf larvae $\left(t_{18}=\right.$ $3093.031, p=0.007$, Cohen's $d=1.362)$, but not in 14-dpf subjects $\left(t_{18}=0.695, p=0.496\right.$, Cohen's $310 d=0.311)$ or in 21 -dpf subjects $\left(t_{18}=0.635, p=0.533\right.$, Cohen's $\left.d=0.286\right)$.

311 A separate analysis of the three ages showed that 14-dpf subjects had a significant preference for 312 the novel stimulus $\left(61.10 \pm 22.44 \% ; t_{19}=2.212, p=0.039\right.$, Cohen's $\left.d=0.494\right)$, whereas 7 -dpf $313\left(53.69 \pm 25.47 \% ; t_{19}=0.684, p=0.525\right.$, Cohen's $\left.d=0.145\right)$ and $21-$ dpf subjects $(53.93 \pm$ $31417.23 \% ; t_{19}=0.733, p=0.472$, Cohen's $\left.d=0.164\right)$ did not.

315

316

\section{Experiment 3: Recognition of Bi-Dimensional Geometrical Figures}

317 In the test phase, subjects, as a whole, spent $303.04 \pm 110.65 \mathrm{~s}(42.09 \pm 15.37 \%)$ close to one or 318 the other stimulus with a significant difference amongst ages $\left(F_{2,56}=14.482, p<0.001, \eta p^{2}=\right.$ 319 0.341). A linear trend analysis showed that time spent close to the stimuli significantly decreased 320 with age $(p<0.001)$. Larvae did not show a preference for the familiar or the novel stimulus 321 (percentage time spent close the novel stimulus: $51.56 \pm 9.94 \%$; $t_{58}=1.205, p=0.233$, Cohen's $322 d=0.157$; Fig. $3 \mathrm{C}$ ). The ANOVA on percentage time spent close to the familiar stimulus found a 323 significant effect of age $\left(F_{2,53}=3.691, p=0.032, \eta \mathrm{p}^{2}=0.122\right)$. There was no significant effect of 324 stimulus used for familiarization $\left(F_{1,53}=1.940, p=0.169, \eta p^{2}=0.035\right)$ nor significant 325 interaction $\left(F_{2,54}=0.479, p=0.622, \eta p^{2}=0.017\right)$.

326 A separate analysis of the three ages showed that 14-dpf subjects had a significant preference for 327 the novel stimulus $\left(53.61 \pm 7.08 \%, t_{18}=2.224, p=0.039\right.$, Cohen's $\left.d=0.510\right)$. Seven-dpf and 21328 dpf subjects did not show significant preference (7-dpf: $54.32 \pm 10.16 \%, t_{19}=1.902, p=0.073$, 329 Cohen's $d=0.425 ; 21$-dpf: $46.85 \pm 10.74 \%, t_{19}=1.313, p=0.205$, Cohen's $d=0.294$ ). 
330 However, the effect size and the $\mathrm{p}$ value close to the threshold for statistical significance suggest 331 that the 7-dpf subjects might have showed a trend of preference similar to that of 14-dpf subjects. 332

333 Meta-analysis of the three NOR experiments

334 1) Overall analysis of the three ages

335 Despite the presence of minor differences, the three experiments essentially measured the same 336 parameter (i.e., the tendency to share time between a familiar object and a novel one). We

337 performed a global analysis of the three NORt experiments at the three ages.

338 Time spent near the stimuli. Larvae showed a general tendency to decrease the time close to the 339 stimuli with age $\left(F_{2,169}=13.970, p<0.001, \eta p^{2}=0.142\right.$; linear trend: $p=0.036$; Fig. 4). There

340 was a significant difference amongst the three experiments $\left(F_{2,169}=64.078, p<0.001, \eta p^{2}=\right.$

$3410.431)$ and interaction $\left(F_{4,169}=3.921, p=0.005, \eta p^{2}=0.085\right)$.

342 Preference for the novel stimulus. Overall larvae showed a preference for novel stimulus (53.15 $343 \pm 20.28 \% ; t_{177}=2.071, p=0.040$, Cohen's $\left.d=0.155\right)$. There was no effect of age $\left(F_{2,69}=1.438\right.$, $\left.344 p=0.240, \eta \mathrm{p}^{2}=0.017\right)$, experiment $\left(F_{2,169}=0.814, p=0.445, \eta \mathrm{p}^{2}=0.010\right)$ or significant 345 interaction $\left(F_{4,169}=0.277, p=0.893, \eta p^{2}=0.007\right)$. 346

347 2) Analysis of 14-dpf larvae

348 Various lines of evidence point to the possibility that only 14-dpf larvae fully responded to the 349 NORt paradigm (see discussion). We performed a joint analysis of the three NORt experiments 350 restricted to larvae of this age.

351 Preference for the novel stimulus. We found a significant preference for the novel stimulus

$352\left(56.31 \pm 22.05 \% ; t_{58}=4.635, p<0.001, \eta p^{2}=0.603\right)$. There was no significant difference in the 
353 preference for the novel stimulus amongst the three experiments $\left(F_{2,56}=0.707, p=0.497, \eta p^{2}=\right.$ $3540.025)$.

355 Effect of stimulus used in the familiarization phase. There was a significant effect of this factor 356 in experiment $1 \mathrm{~b}\left(t_{18}=2.194, p=0.042\right.$, Cohen's $\left.d=0.986\right)$, indicating a spontaneous 357 preference of 14-dpf larvae for the red over the green colour (Fig. 5A). No difference between

358 stimuli was found in the other two experiments (experiment 2: $t_{18}=0.695, p=0.496$, Cohen's $d$ $359=0.311$, Fig. 5B; experiment 3: $t_{17}=1.532, p=0.144$, Cohen's $d=0.704$, Fig. 5C).

360

361

\section{Experiment 4: Development of Neophobia}

362

In the test phase, subjects, as a whole, spent $208.16 \pm 133.31 \mathrm{~s}(34.69 \pm 22.22 \%)$ close to the 363 novel stimulus with a significant difference amongst ages $\left(F_{2,57}=3.198, p=0.048, \eta p^{2}=0.101\right.$;

364 Fig. 6A). A significant linear trend $(p=0.015)$ indicates that the proportion of time close to the stimulus decreased with increasing age. The proportion of time close to the stimulus increased throughout the test ("minutes of the test": $\chi^{2}{ }_{1}=34.973, p<0.001, \eta p^{2}=0.061$; linear trend: $p<$ $0.001)$ with a significant difference amongst ages $\left(\chi_{2}^{2}=6.341, p=0.042, \eta p^{2}=0.014\right)$, but not the interaction $\left(\chi_{2}^{2}=4.814, p=0.090, \eta p^{2}=0.009\right)$. A separate linear trend analysis for the three age groups shows that larvae of all the three ages significantly decreased their neophobia during the test (7 dpf: $p=0.004 ; 14$ dpf: $p=0.049 ; 21 \mathrm{dpf:} p<0.001$; Fig. 6B, C, D).

371

\section{DISCUSSION}

373 To assess the presence of recognition memory in zebrafish larvae, we adapted the most used

374 paradigm in this field, the Novel Object Recognition tests (NORt), a procedure that exploits the 375 tendency of most vertebrates to explore objects they have never seen before (Ennaceur, 2010). 
376 We observed an overall tendency of larvae to spend more time in the vicinity of the novel

377 compared to the familiar stimulus, indicating that recognition memory likely emerges in

378 zebrafish from the first weeks of life. However, the preference appears to be fully significant

379 only in the 14-dpf larvae and in two out of three recognition experiments, suggesting that

380 subjects' age and type of stimuli may affect memory assessment.

381 The non-linear effect of age in the recognition memory experiments (i.e., 14-dpf larvae $>7$-dpf

382 larvae $=21-$ dpf larvae) was likely due to two concomitant causes. The first is an ontogenetic

383 change in the propensity to approach a novel object, which is commonly observed in many

384 species because of experience, maturation or age-specific variation in ecology (Kendal, Coe \&

385 Laland, 2005; Miller et al., 2015). Often, young individuals tend to explore all new objects, and

386 neophobic response increases as they grow older (Menzel, 1966; Biondi, Bó \& Vassallo, 2010).

387 Researchers have suggested that animals begin their life with almost no information about their

388 environment and that the potential benefits of exploring new objects are high (Shettleworth,

389 2010); such benefits decrease, as the experience accumulated and the costs associated with

390 approaching unfamiliar objects may prevail in older individuals (Greenberg \& Mettke-Hofmann,

391 2001). As shown by experiment 4, this latter pattern seems to characterize zebrafish as well.

392 When exposed to an unfamiliar object in an arena, larvae increased their neophobia with age and

393 time spent in close vicinity of the unfamiliar object by 21-dpf larvae as almost half that spent by

394 7-dpf larvae. Therefore, it is possible that, in our experiments, 21-dpf larvae do discriminate the

395 novel from the familiar stimulus, but their exploration tendency was counterbalanced by an

396 increasing neophobia that hindered the detection of recognition memory. In other species, the

397 neophobic reaction may prevail. Miletto Petrazzini and colleagues (2012), testing 5-day old

398 guppies, observed an initial neophobic response to a novel object introduced in their home tank. 
399 Tested with a NOR procedure, young guppies spent significantly more time near a familiar 400 object than a novel one. The finding that 21-dpf larvae, as well as larvae from the other age 401 groups, showed a neophobic response toward an unfamiliar object in experiment 4 may reinforce 402 the idea that they possess some form of recognition memory. Various authors have observed that 403 to recognize that an object is new, an animal needs to recall features of previously encountered 404 objects (Hughes, 2007; Greggor, Thornton \& Clayton 2015). However, it is difficult to 405 demonstrate this involvement of recognition memory in experiment 4. Subjects did not 406 experience objects before the test and, without the control stimulus, it cannot be excluded that 407 they showed a generalized neophobia against any stimulus.

408 The second possible cause of the age effect is the development of neural circuits that support 409 recognition memory or visual discrimination in general. Differing from many other species of 410 fish, the zebrafish shows a very rapid embryonic development with only three days occurring 411 from fertilization to hatching. Therefore, at birth, the brain of zebrafish larvae is in a very 412 immature stage of development (Nusslein-Volhard \& Dahm, 2002). Larvae start to feed 413 autonomously only at $6 \mathrm{dpf}$, whereas more complex functions such as sociality appear much later 414 in development (Robert, Bill \& Glanzman, 2013; Dreost et al., 2015). The poor response of 7415 dpf larvae to the recognition memory tests may derive from the fact that neural structures crucial 416 to visual discrimination and recognition memory are relatively undeveloped or developed in 417 some individuals but not in others. This cognitive effect remains to be addressed because to date 418 there a no other tests available to measure recognition memory in zebrafish larvae.

419 The difference between recognition experiments was likely due to the presence of innate 420 preference/avoidance towards some of the object's features. Biases in novelty responses have 421 been documented in a variety of organisms (Fantz, 1957; Dorosheva, Yakovlev \& Reznikova, 
422 2011) and could intuitively affect a measure of recognition memory based on the relative

423 preference in approaching two objects. This factor could be particularly important in our study

424 given the early age of the subjects and their poor perceptual experience, due to the maintenance

425 in a bare petri dish as required by the standard laboratory housing conditions for zebrafish larvae.

426 The influence of such a factor is evident in experiment $1 \mathrm{~b}$. Larvae familiarized to green cubes

427 tend to prefer the novel colour (red cube), whereas larvae familiarized to red cubes show a

428 preference for the familiar colour (red cube). Since colour preferences were previously observed

429 in both adults and larval zebrafish (Oliveira et al., 2015; Peeters, Moeskops \& Veenvliet, 2016),

430 before the experiment, we had assessed the colour preference in larvae. Fish of all three ages

431 showed a consistent attraction to the blue colour, whereas the other three colours (green, red and

432 yellow) seemed to be similarly preferred. In light of the results of experiment $1 \mathrm{~b}$, it is likely that

433 larvae have an innate preference of red over green and that this preference was masked in

434 experiment 1a by the strong attraction to blue. Other studies have suggested another

435 methodological factor that is critical for recognition memory and could explain the difference

436 between experiments in our study, that is the similarity between stimuli (e.g., Bettis \& Jacobs,

437 2012). Yet, it seems difficult to attribute our results to this factor. Indeed, we found no evidence

438 of recognition in the experiment with colour stimuli, but in the preference test of experiment 1a,

439 zebrafish proved able to distinguish between colours. It remains to be addressed whether the use

440 of objects and images that differ more would improve the recognition performance of the

441 younger group of zebrafish (7 dpf).

442 Overall, our results confirm that recognition memory can be assessed in zebrafish larvae,

443 provided that subjects' age and type of stimuli are carefully evaluated. This conclusion is

444 consistent with research on other species. The NORt has generally been considered a robust test 
445 to measure recognition memory in rodent species (Antunes \& Baila, 2012). However, researchers

446 also reported various limitations, mainly due to the influence of non-cognitive factors (Ennaceur,

447 2010). For example, besides memory, the NORt is likely affected by the individual propensity to

448 approach a novel object, which affects the amount of information about the objects acquired

449 during familiarization, as well as the measure of preference in the test phase (Akkerman et al.,

450 2012). Several methodological details, such as trial duration and previous experience of the

451 subjects, might also affect the NORt's results (Dere, Huston \& Silva, 2007). The results of fish

452 experiments have revealed similar contrasting effects. For example, a recent study on sex

453 differences in guppies found that males explored the novel object at the beginning of the

454 experiment, whereas females did so at the end (Lucon-Xiccato \& Dadda, 2016). According to the

455 temporal windows considered, one sex or the other would appear to perform more, but an overall

456 analysis revealed no sex difference in recognition memory. Contrary to other studies (Braida et

457 al., 2014; Lucon-Xiccato \& Dadda 2014; Oliveira et al., 2015), May and colleagues (2016)

458 found that zebrafish preferentially approached familiar over novel objects and that this response

459 was further modulated by the size of the objects.

460 The zebrafish is rapidly gaining ground as a model for brain diseases due to great ease in

461 dissecting the genetic and physiological basis of these pathologies very early, in some cases even

462 during embryonic development or in the first days of life (Buckley, Goldsmith \& Franklin, 2008;

463 Leung et al., 2013; Paquet et al., 2009; Spence et al., 2008). Although improvements are still

464 needed, the recognition memory task that we developed may provide an important tool to assess

465 early cognitive functioning zebrafish. Researchers on neuropathologies could enjoy the

466 advantage of detecting memory dysfunctions in their subjects at the age of 14 dpf without

467 waiting to test adult fish (Lucon-Xiccato \& Dadda, 2014; May et al., 2016). In addition, many 
468 mutagenic lines show high mortality, reducing the number of available adult subjects. The 469 simplicity and low costs of the NORt for larvae may allow using this method as an initial 470 screening of large populations. An additional point of interest regards animal welfare because the 471 NORt is based on spontaneous behaviour and does not require harmful manipulations.

472

\section{CONCLUSIONS}

474 We investigated the presence of recognition memory, the developmental evolution of this ability, 475 and the use of a novel object recognition procedure to measure it in zebrafish larvae. Although 476 our experiments generally suggest that zebrafish larvae already possess some form of recognition 477 memory and that this can be measured at $14 \mathrm{dpf}$, we demonstrated that NOR tests have some 478 limitations in assessing it. In fact, at least in the version developed for rats, this test seems 479 influenced by non-cognitive factors, such as neophobia, previous experience and spontaneous 480 preferences. Therefore, we need more studies pursuing the objective of devising simple 481 procedures to measure recognition memory in zebrafish larvae.

482

483 ACKNOWLEDGEMENTS

484 The authors would like to thank Carola Zoboli, Lorenzo Esposito, Jessica Gatto, and Margot 485 Carli for their help in testing the animals, and Stefano Massaccesi from Department of General 486 Psychology for his help in building the experimental apparatus. This work was carried out within 487 the scope of the project "use-inspired basic research", for which the Department of General 488 Psychology of the University of Padova has been recognized as "Dipartimento di eccellenza" by 489 the Ministry of University and Research (MIUR). 


\section{REFERENCES}

492 Aizenberg M, Schuman EM. 2011. Cerebellar-dependent learning in larval zebrafish. Journal of 493 Neuroscience 31: 8708-8712 DOI: 10.1523/JNEUROSCI.6565-10.2011.

494

495

Akkerman S, Blokland A, Reneerkens O, van Goethem NP, Bollen E, Gijselaers HJM, Lieben 496 CKJ, Steinbusch HWM, Prickaerts J. 2012. Object recognition testing: methodological 497 considerations on exploration and discrimination measures. Behavioural Brain Research 498 232:335-347 DOI: 10.1016/j.bbr.2012.03.022.

499

500

Antunes M, Biala G. 2012. The novel object recognition memory: neurobiology, test procedure, 501 and its modifications. Cogntive Processing 13:93-110 DOI: 10.1007/s10339-011-0430-z. 502

503

Avdesh A, Martin-Iverson MT, Mondal A, Chen M, Askraba S, Morgan N, Lardelli M, Groth 504 DM, Verdile G, Martins RN. 2012. Evaluation of color preference in zebrafish for learning and 505 memory. Journal of Alzheimer's Disease 28:459-469 DOI: 10.3233/JAD-2011-110704.

506

507 Bandmann O, Burton EA. 2010. Genetic zebrafish models of neurodegenerative diseases.

508 Neurobiology of disease 40:58-65. DOI: 10.1016/j.nbd.2010.05.017

509

510 Barnes CA, Burke SN, Ryan L. 2012. Characterizing cognitive aging of recognition memory and 511 related processes in animal models and in humans. Frontiers in Aging Neuroscience 4:15 DOI: $512 \quad 10.3389 /$ fnagi.2012.00015. 
514 Bettis T, Jacobs LF. 2012. Sex differences in object recognition are modulated by object

515 similarity. Behavioural Brain Research 233:288-292.

516

517 Biondi LM, Bó MS, Vassallo AI. 2010. Inter-individual and age differences in exploration, 518 neophobia and problem-solving ability in a Neotropical raptor (Milvago chimango). Animal 519 Cognition 13:701-710 DOI: 10.1007/s10071-010-0319-8.

520

521 Bisazza A, Piffer L, Serena G, Agrillo C. 2010. Ontogeny of numerical abilities in fish. Plos One 522 5:e15516 DOI: 10.1371/journal.pone.0015516.

523

524 Blaser R, Heyser C. 2015. Spontaneous object recognition: a promising approach to the 525 comparative study of memory. Frontiers in Behavioral Neuroscience 9:183 DOI:

$52610.3389 /$ fnbeh.2015.00183.

527

528 Braida D, Donzelli A, Martucci R, Ponzoni L, Pauletti A, Langus A, Sala, M. 2013. Mice 529 discriminate between stationary and moving 2D shapes: Application to the object recognition 530 task to increase attention. Behavioural Brain Research 242:95-101. DOI:

$531 \quad 10.1016 /$ j.bbr.2012.12.040.

532

533 Braida D, Ponzoni L, Martucci R, Sala M. 2014. A new model to study visual attention in 534 zebrafish. Progress in Neuro-Psychopharmacology and Biological Psychiatry 55:80-86 DOI: 535 10.1016/j.pnpbp.2014.03.010. 
537 Buckley CE, Goldsmith P, Franklin RJ. 2008. Zebrafish myelination: a transparent model for

538 remyelination?. Disease Models \& Mechanisms 1:221-228 DOI: 10.1242/dmm.001248.

539

540 Dere E, Huston JP, Silva MADS. 2007. The pharmacology, neuroanatomy and neurogenetics of 541 one-trial object recognition in rodents. Neuroscience \& Biobehavioral Reviews 31:673-704 DOI:

542 10.1016/j.neubiorev.2007.01.005.

543

544 Dorosheva EA, Yakovlev IK, Reznikova ZI. 2011. An innate template for enemy recognition in 545 red wood ants. Entomological Review 91;274-280 DOI: 10.1134/S0013873811020151.

546

547 Dreosti E, Lopes G, Kampff AR, Wilson SW. 2015. Development of social behavior in young 548 zebrafish. Frontiers in Neural Circuits 9:39 DOI: 10.3389/fncir.2015.00039.

549

550 Ennaceur A. 2010. One-trial object recognition memory in rats and mice: methodological and 551 theoretical issues. Behavioural Brain Research 215:244-54 DOI: 10.1016/j.bbr.2009.12.036.

552

553 Ennaceur A, Delacour J. 1988. A new one-trial test for neurobiological studies of memory in 554 rats. 1: Behavioral data. Behavioural Brain Research 31:47-59 DOI: 10.1016/0166555 4328(88)90157-X.

556

557 Fantz RL. 1957. Form preferences in newly hatched chicks. Journal of Comparative and 558 Physiological Psychology 50 :422 DOI: 10.1037/h0044973. 
560 Gerlai R. 2016. Learning and memory in zebrafish (Danio rerio). In: Detrich III W, Westerfield

561 M, Zon LI, eds. Methods Cell Biology. Cambridge: Academic Press, 134:551-586 DOI:

$562 \quad 10.1016 /$ bs.mcb.2016.02.005

563

564 Greenberg R, Mettke-Hofmann C. 2001. Ecological aspects of neophobia and neophilia in birds.

565 In: Val Nolan Jr V, Thompson CF, eds. Current Ornithology. Boston: Springer, 16:119-178

566 DOI: $10.1007 / 978-1-4615-1211-0 \_3$

567

568 Greggor AL, Thornton A, Clayton NS. 2015. Neophobia is not only avoidance: improving 569 neophobia tests by combining cognition and ecology. Current Opinion in Behavioral Sciences 6: 570 82-89. DOI: 10.1016/j.cobeha.2015.10.007.

571

572 Hamilton TJ, Myggland A, Duperreault E, May Z, Gallup J, Powell RA, Schalomon M, Digweed 573 SM. 2016. Episodic-like memory in zebrafish. Animal Cognition 19:1071-1079 DOI:

$574 \quad 10.1007 / \mathrm{s} 10071-016-1014-1$

575

576 Hinz RC, de Polavieja GG. 2017. Ontogeny of collective behavior reveals a simple attraction 577 rule. Proceedings of the National Academy of Sciences of the United States of America 578 114:2295-2300 DOI: 10.1073/pnas.1616926114.

579

580 Huang YY, Neuhauss SC. 2008. The optokinetic response in zebrafish and its applications.

581 Frontiers in Bioscience 13:1899-1916.

582 
583 Hughes RN. 2007. Neotic preferences in laboratory rodents: issues, assessment and substrates.

584 Neuroscience \& Biobehavioral Reviews 31:441-464. DOI: 10.1016/j.neubiorev.2006.11.004.

585

586 Johnson MH, Horn G. 1986. Dissociation of recognition memory and associative learning by a 587 restricted lesion of the chick forebrain. Neuropsychologia 24:329-340 DOI: 10.1016/0028$588 \quad 3932(86) 90018-7$.

589

590 Kendal RL, Coe RL, Laland KN. 2005. Age differences in neophilia, exploration, and innovation 591 in family groups of callitrichid monkeys. American Journal of Primatology 66:167-188 DOI: 592 10.1002/ajp.20136.

593

594 Kornum BR, Thygesen KS, Nielsen TR, Knudsen GM, Lind NM. 2007. The effect of the inter595 phase delay interval in the spontaneous object recognition test for pigs. Behavioural Brain 596 Research 181:210-217 DOI: 10.1016/j.bbr.2007.04.007.

597

598 Leung LC, Wang GX, Mourrain P. 2013. Imaging zebrafish neural circuitry from whole brain to 599 synapse. Frontiers in Neural Circuits 7:76. DOI: 10.3389/fncir.2013.00076.

600

601 Lucon-Xiccato T, Dadda M. 2014. Assessing memory in zebrafish using the one-trial test.

602 Behavioural processes 106:1-4 DOI: 10.1016/j.beproc.2014.03.010.

603

604 Lucon-Xiccato T, Dadda M. 2016. Guppies show behavioural but not cognitive sex differences 605 in a novel object recognition test. Plos One 11:e0156589 DOI: 10.1371/journal.pone.0156589. 
606

607 Magurran AE, Seghers BH. 1990. Population differences in the schooling behaviour of newborn 608 guppies, Poecilia reticulata. Ethology 84:334-342 DOI: 10.1111/j.1439-0310.1990.tb00807.x. 609

610 May Z, Morrill A, Holcombe A, Johnston T, Gallup J, Fouad K, Schalomon M, Hamilton TJ. 611 2016. Object recognition memory in zebrafish. Behavioural Brain Research 296 :199-210 DOI: $612 \quad 10.1016 /$ j.bbr.2015.09.016.

613

614 Menzel EW. 1966. Responsiveness to objects in free-ranging Japanese monkeys. Behaviour 615 26:130-150 DOI: 10.1163/156853966X00065.

616

617 Miletto Petrazzini ME, Agrillo C, Piffer L, Dadda M, Bisazza A. 2012. Development and 618 application of a new method to investigate cognition in newborn guppies. Behavioural Brain 619 Research 233:443-449 DOI: 10.1016/j.bbr.2012.05.044.

620

621 Miller R, Bugnyar T, Pölzl K, Schwab C. 2015. Differences in exploration behaviour in common 622 ravens and carrion crows during development and across social context. Behavioral Ecology and 623 Sociobiology 69:1209-1220 DOI: 10.1007/s00265-015-1935-8.

624

625 Nusslein-Volhard C, Dahm R. 2002. Zebrafish. Oxford: Oxford University Press.

626

627 Norton WHJ. 2013. Toward developmental models of psychiatric disorders in zebrafish.

628 Frontiers in Neural Circuits 7:79 DOI: 10.3389/fncir.2013.00079. 
629

630 Oliveira J, Silveira M, Chacon D, Luchiari A. 2015. The zebrafish world of colors and shapes:

631 preference and discrimination. Zebrafish 12:166-173 DOI: 10.1089/zeb.2014.1019.

632

633 Paquet D, Bhat R, Sydow A, Mandelkow EM, Berg S, Hellberg S, Fälting J, Distel M, Köster

634 RW, Schmid B, Haass C. 2009. A zebrafish model of tauopathy allows in vivo imaging of

635 neuronal cell death and drug evaluation. The Journal of Clinical Investigation 119:1382-1395

636 DOI: $10.1172 / \mathrm{JCI} 37537$.

637

638 Park JS, Ryu JH, Choi TI, Bae YK, Lee S, Kang HJ, Kim CH. 2016. Innate color preference of 639 zebrafish and its use in behavioral analyses. Molecules and Cells 39:750-755 DOI:

$640 \quad 10.14348 /$ molcells.2016.0173.

641

642 Pascalis O. 1994. Recognition memory in 3-to 4-day-old human neonates. Neuroreport 5:1721-

6431724 DOI: 10.1097/00001756-199409080-00008.

644

645 Peeters BWMM, Moeskops M, Veenvliet ARJ. 2016. Color preference in Danio rerio: effects of 646 age and anxiolytic treatments. Zebrafish 13:330-334 DOI: 10.1089/zeb.2015.1150.

647

648 Piffer L, Miletto Petrazzini ME, Agrillo C. 2013. Large number discrimination in newborn fish. 649 Plos One 8:e62466 DOI: 10.1371/journal.pone.0062466.

650 
651 Reger ML, Hovda DA, Giza CC. 2009. Ontogeny of rat recognition memory measured by the 652 novel object recognition task. Developmental Psychobiology 51:672-678 DOI:

653 10.1002/dev.20402.

654

655 Richendrfer H, Pelkowski SD, Colwill RM, Creton R. 2012. On the edge: pharmacological 656 evidence for anxiety-related behavior in zebrafish larvae. Behavioural Brain Research 228:99657 106. DOI: $10.1016 /$ j.bbr.2011.11.041.

658

659 Roberts AC, Bill BR, Glanzman DL. 2013. Learning and memory in zebrafish larvae. Frontiers 660 in Neural Circuits 7:126 DOI: 10.3389/fncir.2013.00126.

661

662 Sager JJ, Bai Q, Burton EA. 2010. Transgenic zebrafish models of neurodegenerative diseases. 663 Brain Structure and Function 214:285-302. DOI: 10.1007/s00429-009-0237-1.

664

665 Sakai C, Ijaz S, Hoffman EJ. 2018. Zebrafish models of neurodevelopmental disorders: past, 666 present, and future. Frontiers in molecular neuroscience 11:294. DOI:

$667 \quad 10.3389 /$ fnmol.2018.00294.

668

669 Shettleworth SJ. 2010. Clever animals and killjoy explanations in comparative psychology.

670 Trends in cognitive sciences 14:477-481 DOI: 10.1016/j.tics.2010.07.002.

671

672 Soto FA, Wasserman EA. 2014. Mechanisms of object recognition: what we have learned from 673 pigeons. Frontiers in Neural Circuits 8:122 DOI: 10.3389/fncir.2014.00122. 
674

675 Spence R, Gerlach G, Lawrence C, Smith C. 2008. The behaviour and ecology of the zebrafish, 676 Danio rerio. Biological Reviews 83:13-34 DOI: 10.1111/j.1469-185X.2007.00030.x.

677

678 Valente A, Huang KH, Portugues R, Engert F. 2012. Ontogeny of classical and operant learning 679 behaviors in zebrafish. Learning \& Memory 19:170-177 DOI: 10.1101/1m.025668.112.

680

681 Wilson C. 2012. Aspects of larval rearing. ILAR Journal 53:169-178 DOI: 10.1093/ilar.53.2.169. 


\section{Figure 1}

\section{Experimental apparatuses}

Top view of the experimental apparatuses used in this study. (A) Experiment la (spontaneous colour preference). Larvae were observed in a petri dish subdivided into four equal sectors. (B) Experiment 1b (NOR test). Larvae were familiarized to two objects of the same colour and tested with one familiar object and one of a different colour. (C) Experiment 2 (NOR test). Larvae were familiarized to two objects of the same shape and tested with one familiar object and one of a different shape. (D) Experiment 3 (NOR test). Larvae were collectively familiarized to one printed geometric figure and then individually tested in a rectangular arena with one familiar geometric figure and a novel one. (E) Experiment 4 (neophobic response test). Larvae were placed in a rectangular arena containing an unfamiliar object (a black cone), then we measured the time spent in the vicinity of the object. 
B

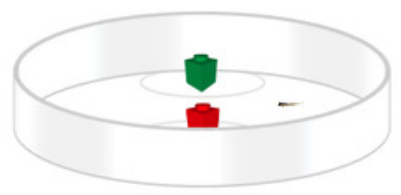

A

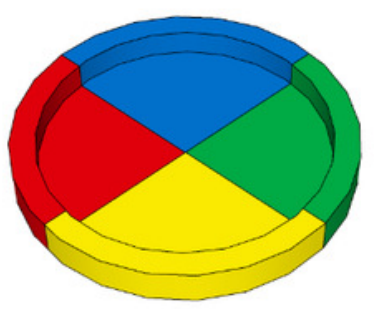

C
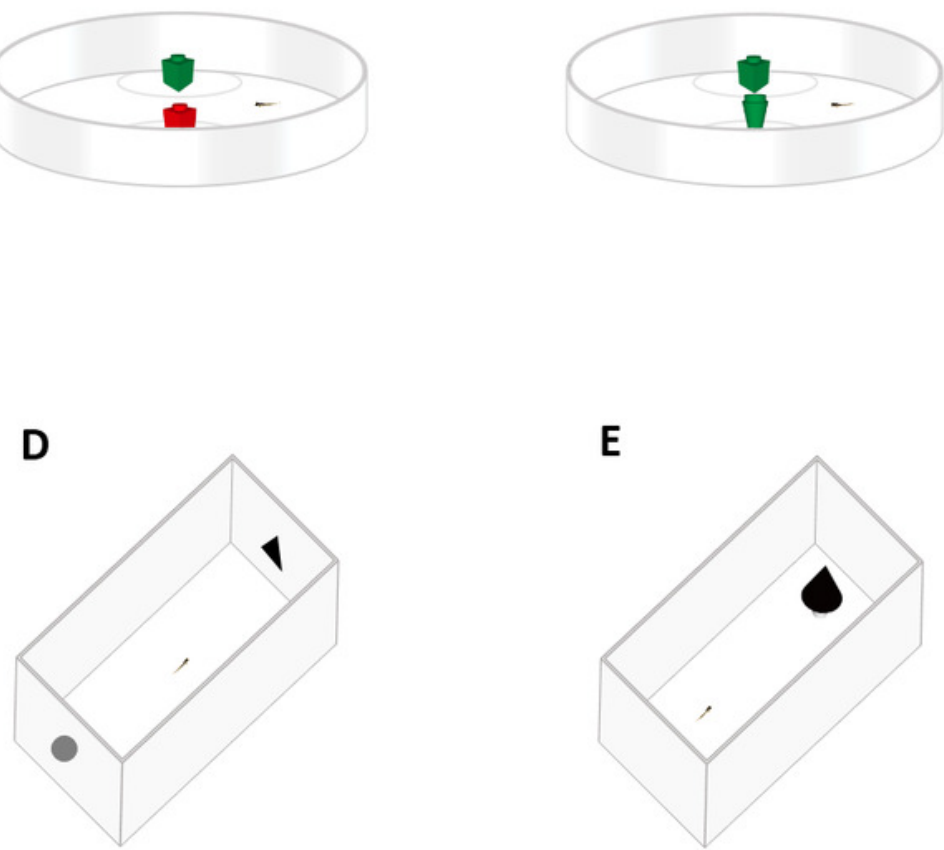

E

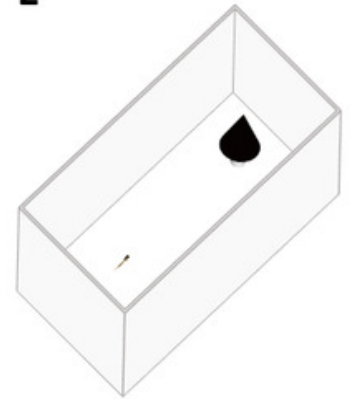




\section{Figure 2}

Colour preference in larvae

(A) Percentage of time (mean \pm standard error) spent in each coloured sector in 7, 14 and 21-dpf larvae. Larvae showed a preference for the blue sectors compared to the other colours (all $\mathrm{P}<0.001$ ). There was no difference amongst the three ages in the time spent in the four sectors $(P=0.705)$. (B, $\mathbf{C}, \mathbf{D})$ Temporal pattern of time spent in the four sectors for each age. The preference for blue decreased during the trial $(P<0.001)$. Dotted lines represented the expected proportion of time in each sector by chance (25\%).
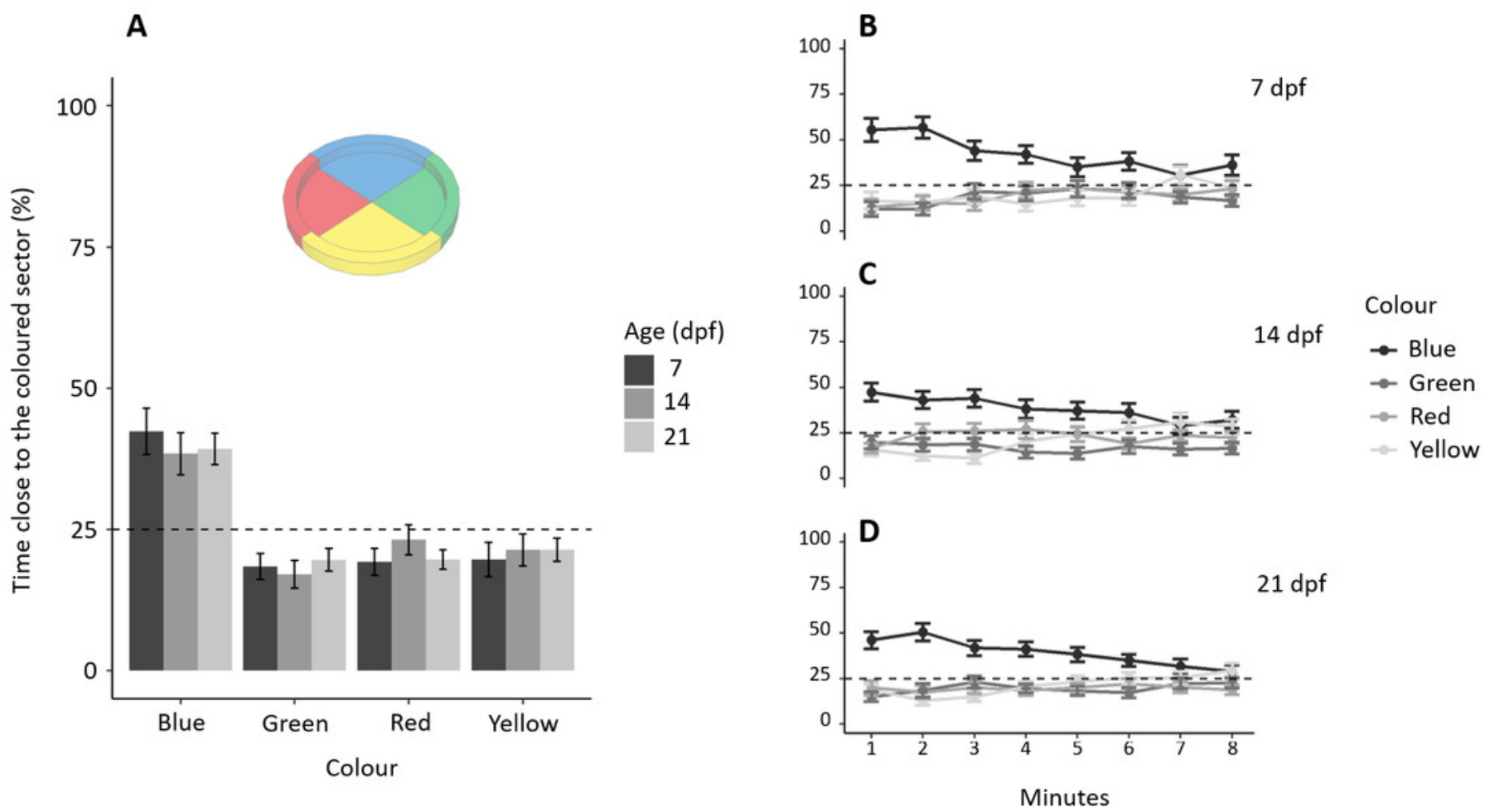


\section{Figure 3}

Percentage of time (mean \pm standard error) close to the novel stimulus in the tree NORt experiments.

(A) Memory for object's colour. Larvae did not show a preference for the familiar or the novel stimulus ( $P=0.559)$, and there was no difference amongst the ages $(P=0.884)$. (B) Memory for object's shape. Larvae showed an overall preference for the novel stimulus $(P=0.043)$, and there was no difference amongst the ages $(P=0.389)$. (C) Memory for the shape of a bidimensional geometrical figure. Larvae did not show an overall preference for the familiar or the novel stimulus $(P=0.233)$, but the three ages showed a significant difference in preference $(P=0.032)$. Dotted lines represented the expected proportion of time by chance $(50 \%)$ and asterisks indicated significant differences from chance $(P<0.05)$.

A

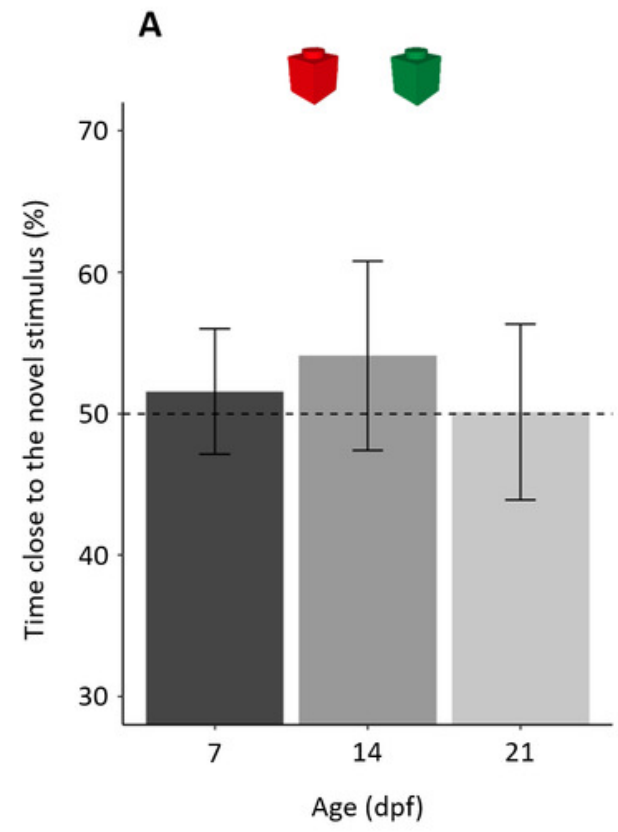

B

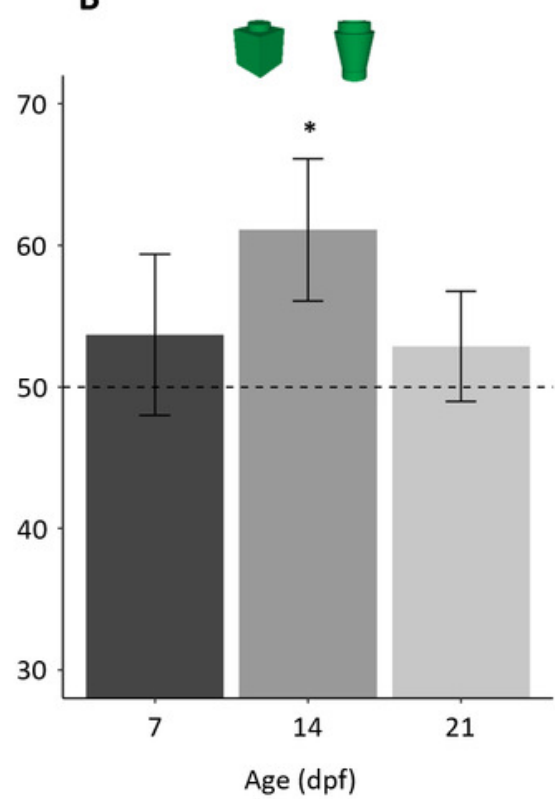

C

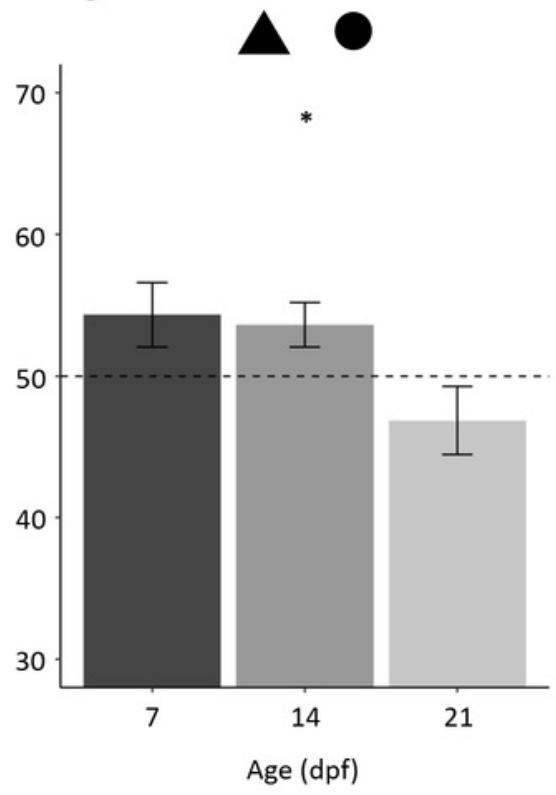




\section{Figure 4}

Time (mean \pm standard error) spent close to both stimuli in the three NORt experiments.

Overall time close to the stimuli decreased with age $(P<0.001$; linear trend: $P=0.036)$ with a significant difference amongst experiments $(P<0.001)$ and interaction

A

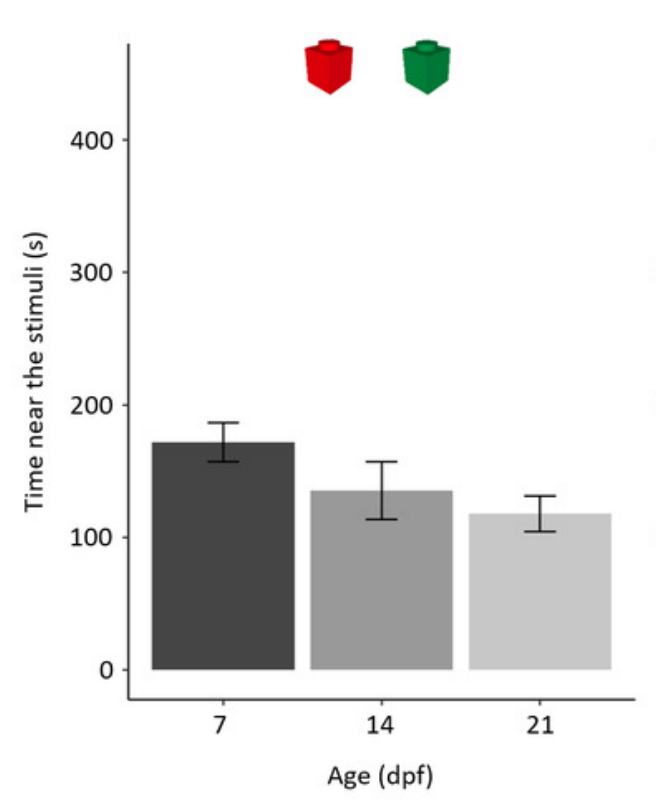

B

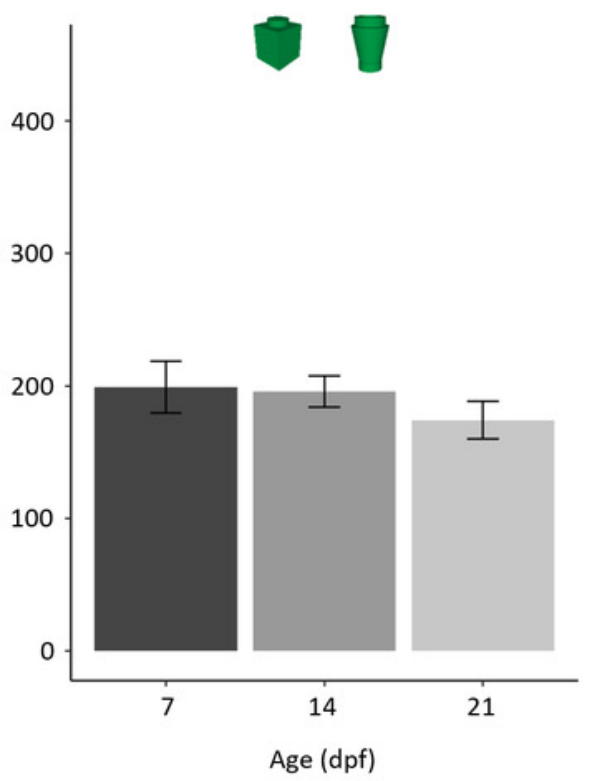

C

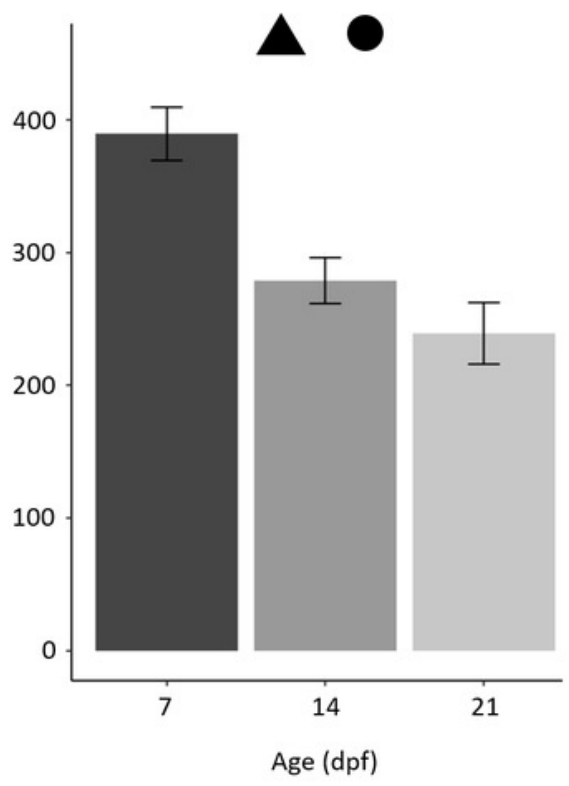




\section{Figure 5}

Percentage of time (mean \pm standard error) close to the novel stimulus in 14-dpf larvae in relation to the stimulus used in the familiarization phase.

(A) Subjects showed a spontaneous preference for the red colour, and they tended to prefer the red colour for both familiar and novel objects. (B), (C) No difference was found in the two other NORt experiments. Dotted lines represented the expected proportion of time by chance (50\%) and asterisks indicated significant differences between the two conditions ( $P<0.05)$.

A

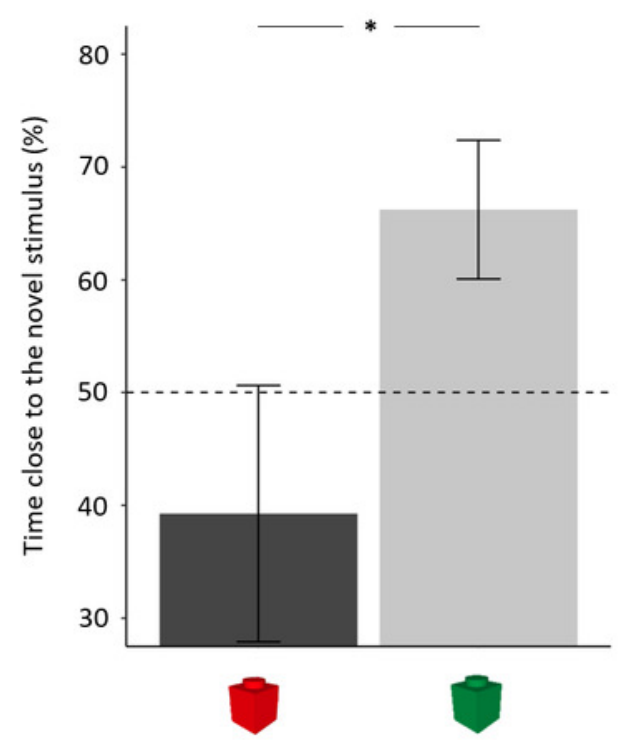

Stimuli during familiarization
B

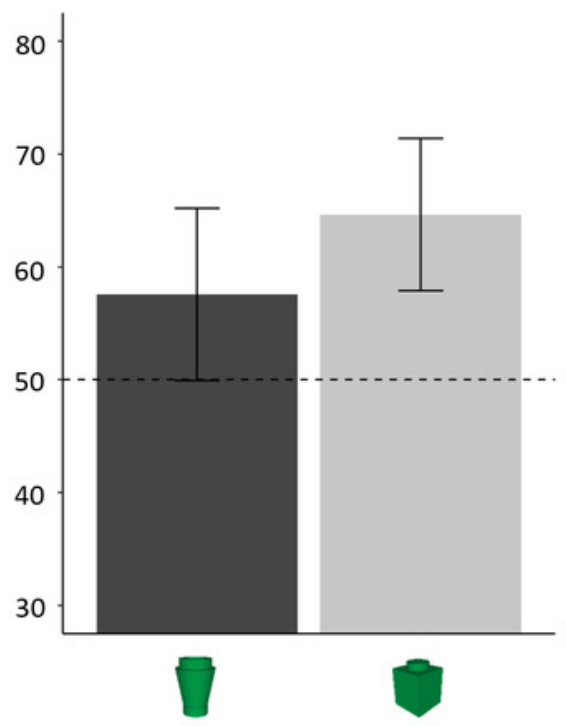

Stimuli during familiarization
C

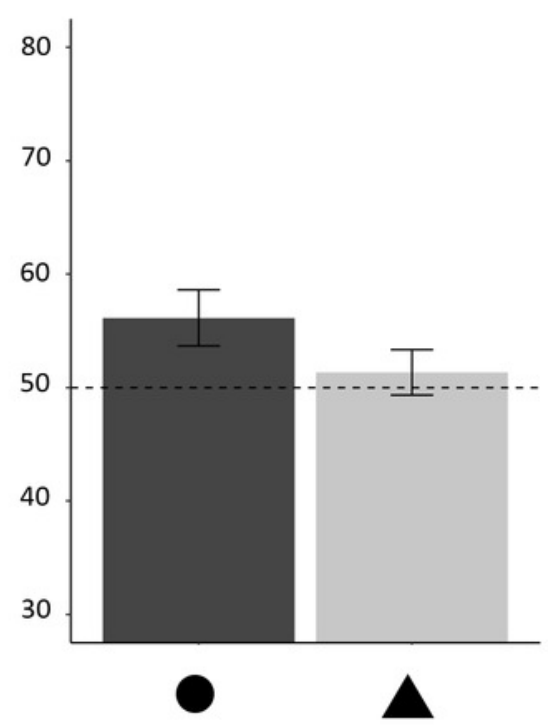

Stimuli during familiarization 


\section{Figure 6}

Development of neophobia in larvae.

(A) Time near a new stimulus decreases with increasing age $(P=0.048$; linear trend: $P<$ 0.001). (B, C, D) Tendency to approach the stimulus for each age increased throughout the test $(P<0.001)$. Dotted lines represented the expected proportion of time by chance $(25 \%)$.
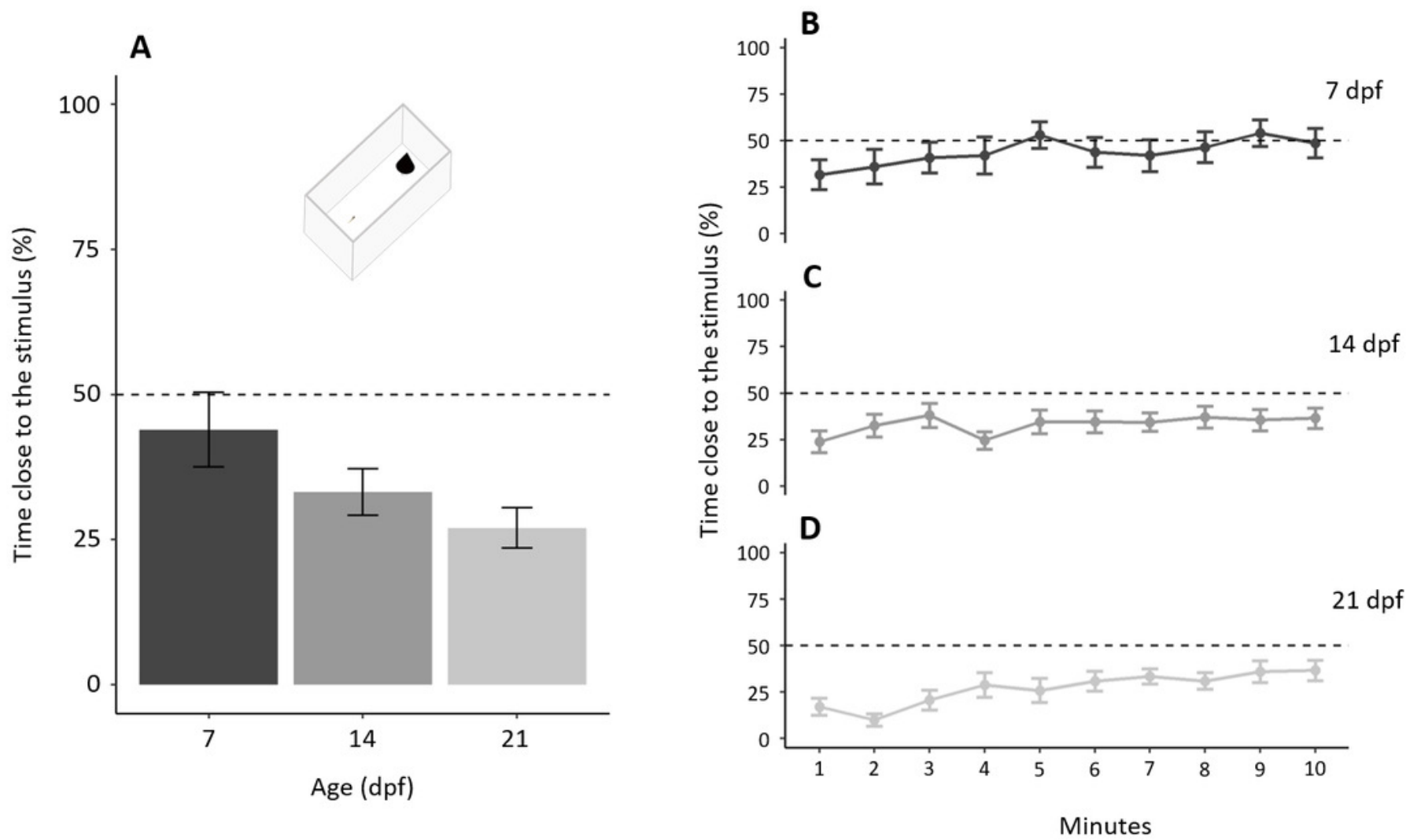